\title{
Retrospective Analyses of 200 Patients Over 65 Years Receiving Albumin Treatment in Intensive Care Unit
}

\author{
Banu CEVIK, Yucel YUCE* \\ University of Health Sciences Kartal Dr. Lutfi Kirdar Education and Research Hospital, Anaesthesiology and Reanimation Department, \\ Kartal, Istanbul, Turkey \\ *Corresponding author: dryyuce@gmail.com
}

\begin{abstract}
The aim of this study is to evaluate the effectiveness of albumin infusion in critically ill geriatric patients and discuss the prognostic impact on mortality. This study is a retrospective analysis of patients with hypoalbuminemia $(\leq 2.5 \mathrm{~g} / \mathrm{dl})$ over 65 years and older followed-up in a 38-bed-mixed medical-surgical tertiary intensive care unit (ICU) of a 650-bed-academic hospital affiliated to University of Health Sciences in İstanbul during 1-year-period. We collected the data including sex, cause of admission, length of stay, duration of mechanical ventilation and APACHE II scores from patients' medical records. The amount of albumin infused per patient and laboratory evaluation concerning albumin levels was processed by hospital electronic database. Overall 200 patients were included study. Among the patients, 90 (45\%) were female, and $110(53.5 \%)$ were male with overall mean age of $78.62 \pm 7.63$ years. The cause of admission was due to non-surgical reasons in 119(59.5\%) patients. Mean length of stay and mechanical ventilation time was significantly higher in non-survivors. The amount of albumin used per patient was similar in both study groups $(\mathrm{p}=0.276)$. Pretreatment level of serum album was $2.15 \pm 0.28 \mathrm{~g} / \mathrm{dl}$ and $2.10 \pm 0.31 \mathrm{~g} / \mathrm{d} \mathrm{l}$ in both groups respectively $(\mathrm{p}=0.364)$ having no significant increase after treatment $(\mathrm{p}=0.352)$. In the non-survivors group, APACHE II score was significantly high. Long-term mortality ratio was significantly high which indicated that albumin had no beneficial effect on long-term mortality. The albumin transfusion in critically ill patients over 65 years old has no considerable effect on the treatment of hypoalbuminemia and ICU mortality.
\end{abstract}

Keywords: albumin, APACHE II, mortality

Cite This Article: Banu CEVIK, and Yucel YUCE, "Retrospective Analyses of 200 Patients Over 65 Years Receiving Albumin Treatment in Intensive Care Unit." American Journal of Clinical Medicine Research, vol. 6, no. 1 (2018): 1-4. doi: 10.12691/ajcmr-6-1-1.

\section{Introduction}

The population of 'older person' aged 60 years and above increased from $9.2 \%$ to $11.7 \%$ from 1990 to 2013 and this ratio is expected to reach to $21.1 \%$ until 2050 [1]. Parallel to the global aging, in Turkey, the proportion of citizens aged over 65 years is expected to reach from $7.5 \%$ to $10.2 \%$ by $2023,21 \%$ by 2050 and $28 \%$ by 2075 [2]. This dramatic increase results in the many geriatric patients requiring hospitalization and critical care settings. Because of the structural and functional impairment of aging, physiological changes may develop in many major organ systems resulted with complex and chronic health problems in these patients. Serum level of albumin is also affected by this negative process other than patient's poor nutritional status.

Albumin is an acute-phase protein synthesized by the liver and responsible for transport of various crucial substances and maintenance of normal plasma colloid oncotic pressure by antithrombotic effect [3]. It binds reversibly with drugs, hormones, bilirubin and metal ions and plays an important role in volume expansion and scavenging of free radicals [4].
Albumin solutions have been used for the treatment of hypoalbuminemia more than 60 years but the use of albumin in critically ill patients is still controversial despite the widely publicized meta-analysis [5]. Especially in patients over 65 years and older, the causes of hypoalbuminemia are multifactorial and the maintenance of normal serum albumin level may be a challenge.

The aim of this study is to evaluate the effectiveness of albumin infusion in critically ill geriatric patients and discuss the prognostic impact on mortality.

\section{Materials and Methods}

This study is a retrospective analysis of patients with hypoalbuminemia $(\leq 2.5 \mathrm{~g} / \mathrm{dl})$ over 65 years and older followed-up in a 38-bed-mixed medical-surgical tertiary intensive care unit (ICU) of a 650-bed-academic hospital affiliated to University of Health Sciences in İstanbul during 1-year-period. Among these patients, a sample of 200 patients was randomly assigned to the study group.

Our ICU is a closed department in which the patients are under the direct care of the staffing by anesthesiologists accepting 1600 patients/year. The institution has no other ICU and all critically ill non-surgical and surgical patients 
except cardiothoracic surgery are managed in this unit. A three-step process was used for collection of data. We initially reviewed the ICU admission registry to find the patients aged over 65 years. After that, we collected the data including sex, cause of admission, length of stay, duration of mechanical ventilation and APACHE II scores from patients' medical records. APACHE II is a severity score which includes 11 routine physiological measurements, Glasgow Coma Score, age, and previous health status. This scoring is used to predict hospital mortality, with higher APACHE II score meaning greater risk [6]. On the third step, the amount of albumin infused per patient and laboratory evaluation concerning albumin levels was processed by hospital electronic database.

Each administration of albumin solution was considered separately in respect of albumin levels of before and after infusion treatment and the correlation of these results were our main objective. The effect of albumin infusion on short-term ( $\leq 10$ days) and long-term ( $\geq 10$ days) mortality rates was another goal of the study.

Chronic health problems during admission were also recorded from patients' medical records and co-morbidities were classified according to the number of major organs affected.

The study was conducted with ethical principles outlined in the Helsinki Declaration and a protocol approved by Scientific Research Ethics Committee of our hospital.

\section{Statistical Analysis}

Statistical analyses were performed using SPSS 20 (SPSS Inc., Chicago, IL, USA). Continuous variables such as age, length of hospital stay, length of MV, APACHE II scores, Pre-and post-treatment serum albumin levels, the amount of albumin used during the treatment were expressed as mean (standard deviation). To investigate the relationship of serum albumin concentration with functional performance and in-hospital mortality rate, multiple logistic regression analysis was used. Results are presented as odds ratios with $95 \%$ confidence interval. Statistical significance was set at $\mathrm{p}<0.05$.

\section{Results}

Overall 200 patients were included study. Among the patients, $90(45 \%)$ were female, and $110(535 \%)$ were male with overall mean age of $78.62 \pm 7.63$ years. The cause of admission was due to non-surgical reasons in $119(\% 59.5)$ patients. Mean length of stay and mechanical ventilation time was significantly higher in non-survivors (Table 1).

The amount of albumin used per patient was similar in both study groups $(p=0.276)$. Pretreatment level of serum album was $2.15 \pm 0.28 \mathrm{~g} / \mathrm{dl}$ and $2.10 \pm 0.31 \mathrm{~g} / \mathrm{dl}$ in both groups respectively $(\mathrm{p}=0.364)$ having no significant increase after treatment $(\mathrm{p}=0.352)$ (Table 2$)$.

In patients with no organ failure or with 1-2 organ failures there was no statistically significant difference in survival, but in patients with 3 and more organ failure mortality was significantly higher. In the non-survivors group, APACHE II score was also significantly high (Table 3).

Long-term mortality ratio was significantly high which indicated that albumin had no beneficial effect on longterm mortality (Table 4).

Table 1. Demographic and clinical characteristics of patients

\begin{tabular}{|l|c|c|c|}
\hline & Survival & Non-suvival & p-values \\
\hline Gender (n, \%) & & & 0.285 \\
Female & $22(11 \%)$ & $68(34 \%)$ & $82(41 \%)$ \\
\hline Male & $28(14 \%)$ & $78.62 \pm 7.63$ & 0.175 \\
\hline Age (year) & $73.53 \pm 6.54$ & $26.6 \pm 38.50$ & $<0.001$ \\
\hline Length of ICU stay (day) & $9.28 \pm 4.54$ & $13(6.5 \%)$ & 0.186 \\
\hline Cause of ICU admission (n, \%) & $68(34 \%)$ & $98(49 \%)$ & $<0.001$ \\
Surgical & $21(10.5 \%)$ & $22.48 \pm 32.31$ & \\
Non-surgical & $10.32 \pm 3.43$ & & \\
\hline Length of MV time (day) & & & \\
\hline
\end{tabular}

Table 2. Outcome of albumin in the study sample

\begin{tabular}{|l|c|c|c|}
\hline & Survival & Non-survival & p-values \\
\hline $\begin{array}{l}\text { Amount of albumin solution (Number of 50 } \\
\text { ml 20\% Albumin solution, mean } \pm \text { SD) }\end{array}$ & $8.62 \pm 6.19$ & $9.73 \pm 7.23$ & 0.276 \\
\hline Pre-transfusion level of albumin (g/dL) & $2.15 \pm 0.28$ & $2.10 \pm 0.31$ & 0.364 \\
\hline Post-transfusion level of albumin (g/dL) & $2.32 \pm 0.35$ & $2.28 \pm 0.32$ & 0.352 \\
\hline
\end{tabular}

Table 3. Correlation of co-morbidity and APACHE II scores in survivors and non-survivors

\begin{tabular}{|l|c|c|c|}
\hline & Survival (n=50) & Non-survival (n=150) & p- value \\
\hline $\begin{array}{l}\text { Comorbidity } \\
\text { (number of organ failure) }\end{array}$ & & & \\
$\mathbf{0 - 1}$ & $20(10 \%)$ & $18(9 \%)$ & 0.256 \\
$\mathbf{2}$ & $17(8 \%)$ & $38(19 \%)$ & 0.154 \\
$\mathbf{3} \leq$ & $13(6 \%)$ & $94(47 \%)$ & $<\mathbf{0 . 0 0 1}$ \\
\hline APACHE II score & $16.13 \pm 4.18$ & $30.32 \pm 10.21$ & $<\mathbf{0 . 0 0 1}$ \\
\hline
\end{tabular}


Table 4. Short-term and long-term mortality rates of the patients

\begin{tabular}{|c|c|c|c|}
\hline & $<\mathbf{1 0}$ days & $\mathbf{2 1 0}$ days & p- value \\
\hline Mortality (n; \%) & $51(34 \%)$ & $99(66 \%)$ & $<0.001$ \\
\hline
\end{tabular}

\section{Discussion}

Albumin is widely used as a resuscitation fluid in heterogeneous groups of critically ill patients but it may not be appropriate in all patients even it may be harmful. In critically ill patients, there is often an inflammatory response resulted in capillary leakage and protein loss including albumin. This increased vascular permeability is a major cause of hypoalbuminemia and usually accepted as a reflection of the severity of underlying disease [7].

The role of albumin treatment is an ongoing issue and The SAFE Study Investigators showed the clinical equivalence of albumin and saline for intravascular volume replacement [8]. The association between hypoalbuminemia and poor prognosis in old patients was well described by Hannan et al. [9] and they reported that low albumin level was associated with increased risk of death especially in old patients. The unclear point was the low albumin levels itself or other accompanying factors contributed to this correlation.

Older people are vulnerable to malnutrition and it's usually neglected. The worldwide prevalence of malnutrition among hospitalized geriatric patients ranges from $12 \%$ to $75 \%$ [10]. These high rates demonstrate the importance of nutritional status in elderly population which adversely affecting various organ functions, immune system and wound healing [11]. Unfortunately, the nutritional status of the patients often becomes significantly more compromised during ICU stay due to many factors resulted in the increased length of stay, hospital cost and long-term mortality [12]. A limitation of the study is that it does not handle the nutritional status of this patient population. Therefore, it is not possible to determine whether refractory posttransfusion albumin level is due malnutrition or not. So, further studies are needed to show the effectiveness of albumin infusion in aspects of general or malnourished geriatric patients. Although albumin is a popular serum marker as the determinant of nutritional status recently it's noted that albumin is good for detecting inflammatory process rather than undernourishment [13].

Although 'age' is the main component of scoring systems many studies showed that age itself did not predict mortality in elderly even in oncology patients $[14,15,16,17]$. 'Age' was not considered as our goal in this study so no classification across age groups was identified. Also, disease-related changes of albumin did not adjust. This is an advantage of this study for evaluation of the heterogeneous group of elderly in different functional status.

Controversies about the positive effect of albumin on mortality rates are going on. In the 1990s, a first meta-analysis from the Cochrane group including 30 randomized clinical trials had indicated that mortality rate was higher in patients with hypovolaemia due to injury or surgery, burns and hypoproteinaemia received albumin solution [18]. Conversely, Wilkes et al did not support this detrimental effect of albumin in patients with trauma or surgery, burns, ascites or high-risk neonates [19]. This study reinforces the relation between albumin treatment and mortality outcomes. Long-term mortality was significantly higher in non-survivors $(p<0.001)$. This means that albumin has no beneficial effect on patients having prolonged length of stay in ICU. But the comparison of these patients with a normoalbuminemic elderly group will provide a more definite acknowledgment.

In fact, albumin is mostly prescribed for nutritional intervention or correction of hypoalbuminemia despite lacking clinical and scientific evidence [20,21]. Our results are parallel with this argument and no increase in serum albumin level was detected with albumin infusion therapy.

Another limitation is that the data are only from a single center and this may not be representative of similar critical care units. We believe that this study would be more valuable if it could be conducted on a multicenter basis. If the number of the patient increases the effects of albumin in a heterogeneous group of elderly may be more definitely ruled out.

At this point, a new question comes to our mind that whether there is an alternative of albumin in maintaining the microcirculation in critically ill patients. Boldt et al [22] studied hydroxyethyl starch (HES) as an alternative to these patients and they found no significant difference between albumin and HES groups about cardiorespiratory and circulatory variables. But cardiac index was higher in the HES group. Long-term infusion therapy resulted with improved splanchnic perfusion. In addition, they reported that inflammatory process continued or worsened in albumin group whereas it was better in HES group due to improved microcirculatory hemodynamic. Our results may suggest these conclusions with the higher long-term mortality ratio indicating that albumin had no beneficial effect on long-term mortality.

As a conclusion, on the basis of data above, the albumin transfusion in critically ill patients over 65 years old has no considerable effect on the treatment of hypoalbuminemia and ICU mortality. Each patient must be considered on an individual basis in respect of necessity of albumin infusion. Further studies are needed to address the treatment modalities of the underlying cause of hypoalbuminemia in critically ill elderly patients.

\section{References}

[1] United Nations (UN) Department of Economic and Social Affairs, Population Division World Population Ageing 2015. http://www.un.org/en/development/desa/population/publication/pd f/ageing/WPA2015_Report.pdf (access date: 27.12.2017).

[2] Turkish Statistical Institute, Population Projections, 2013-2075. http://www.turkstat.gov.tr/PreHaberBultenleri.do?id=15844 (access date: 27.12.2017).

[3] Lyons O, Whelan B, Bennett K, O'Riordan D, Silke B. Serum albumin as an outcome predictor in hospital emergency medical admissions. Eur J Intern Med 2010; 21(1): 17-20.

[4] Hankins J. The role of albumin in fluid balance. Nursing 2007; 37 (12 Pt.1):14-5.

[5] Perel P, Roberts I, Ker K. Colloids versus crystalloids for fluid resuscitation in critically ill patients Cochrane Database Syst Rev. 2013 Feb 28; (2): CD000567. 
[6] Knaus WA, Draper EA, Wagner DP, Zimmerman JE. APACHE II: a severity of disease classification system. Crit Care Med 1985; 13(10): 818-29.

[7] Vincent JL, Russell JA, Jacob M, Martin G, Guidet B, Wernerman J, Ferrer R, McCluskey SA, Gattinoni L. Albumin administration in the acutely ill: what is new and where next? Crit Care 2014; 16; 18(4): 231.

[8] The SAFE Study Investigators. A Comparison of Albumin and Saline for Fluid Resuscitation in the Intensive Care Unit. N Engl J Med 2004; 350: 2247-56.

[9] Hannan JL, Radwany SM, Albanese T. In-hospital mortality in patients older than 60 years with very low albumin levels. J Pain Symptom Manage. 2012; 43(3): 631-7.

[10] Harith S,Shahar S, Yusoff NAM, Kamaruzzaman SB, Hua PPJ. The magnitude of malnutrition among hospitalized elderly patients in University Malaya Medical Center. Health Environ J 2010; 1(2): 64-72.

[11] Sullivan DH, Sun S, Walls RC. Protein-energy undernutrition among elderly hospitalized patients: a prospective study. JAMA 1999; 281 (21): 2013-9.

[12] PE Wischmeyer. Malnutrition in the acutely ill patient: is it more than just protein and energy? S Afr J Clin Nutr 2011; 24(3): S1-S7.

[13] Bharadwaj S, Ginoya S, Tandon P, Gohel TD, Guirguis J, Vallabh H, Jevenn A, Hanouneh I. Malnutrition: laboratory markers vs nutritional assessment. Gastroenterology Report, 4(4), 2016, 272-280.
[14] Onal O, Ozgun G. Comparison of the course and prognosis of geriatric patients admitted to the intensive care unit according to BMI and albumin values. Anesth Pain Med 2016; 6(1): e32509.

[15] Morley JE. Anorexia of aging: Physiologic and pathologic. Am J Clin Nutr 1997; 66(4): 760-73.

[16] Gurlich R, Maruna P, Kalvach Z, Peskova M, Germak J, Frasko R. Colon resection of elderly patients comparison of data from the Czech Republic. Arch Gerontol Geriatr 2005; 41(2): 183-90.

[17] Hoekstra HJ. Cancer surgery in elderly. Eur J Cancer 2001; 37: 235-44.

[18] Cochrane Injuries Group Albumin Reviewers. Human albumin administration in critically ill patients: systematic review of randomised controlled trials. BMJ 1998; 317: 235-40.

[19] Wilkes MM, Navickis RJ. Patient survival after human albumin administration. A meta-analysis of randomized, controlled trials. Ann Intern Med 2001; 135: 149-64.

[20] Caraceni P, Tufoni M, Bonavita ME. Clinical use of albumin. Blood Transfus 2013; 11(4):18-25.

[21] Melinyshyn A, Callum J, Jeschke MC, Cartotto R. Albumin supplementation for hypoalbuminemia following burns: unnecessary and costly! J Burn Care Res. 2013; 34(1): 8-17.

[22] Boldt J, Heesen m, Muller M, Pabsdorf M, hempelmann G. The effects of albumin versus hydroxyethyl starch solution on cardiorespiratory and circulatory variables in critically ill patients. Anesth Analg 1996; 83: 254-61. 